\title{
Assessment of Health Care Quality in Chronic Neurological Diseases: Stroke, Multiple Sclerosis and Epilepsy
}

\author{
Nuray Dayapoglu* and Zümrüt Akgün Şahin \\ Nuray Dayapoglu, Faculty of Health Sciences, Atatürk University, Erzurum, Turkey \\ *Corresponding author: Nuray Dayapoglu, Faculty of Health Sciences, Atatürk University, Erzurum, Turkey, Tel: +90 442231 14 54; E-mail: \\ nuraydayapoglu@hotmail.com
}

Received date: September 23, 2016; Accepted date: December 06, 2016; Published date: December 13, 2016

Copyright: (c) 2016 Dayapoglu N, et al. This is an open-access article distributed under the terms of the Creative Commons Attribution License, which permits unrestricted use, distribution, and reproduction in any medium, provided the original author and source are credited.

\begin{abstract}
Objective: The aim of this study is to assess the quality of care in chronic neurological diseases, and to analyze the correlation between patients' dependency and the quality of care.

Methods: The study is designed as a descriptive and cross-sectional one. It was conducted among 184 patients who had visited neurology clinics of Ataturk University, Turkey, from March 2014 to July 2014. Data was collected by using a Self-made questionnaire, PACIC (Patient Assessment of Chronic Illness Care) and Barthell Index (BI). For data evaluation, percentage distribution, mean, independent samples $t$ test, variance analysis and Pears on correlation analysis were utilized.
\end{abstract}

Results: Overall mean score of PACIC scale was found to be $3.05 \pm 0.57$. The lowest mean score was obtained in follow-up/coordination subscale $(2.73 \pm 0.86)$ among sub-scales of the scale, the highest mean score was found to be in patient activation subscale $(3.20 \pm 0.74)$. The mean score for $\mathrm{BI}$ was $66.60 \pm 38.22$. The mean score of $\mathrm{PACIC}$ varied significantly according to age, marital status, education, disease diagnosis, co morbid conditions and hospitalization duration and hospital experience.

Conclusion: It was found that the satisfaction of patients in terms of chronic disease is at a moderate level, and the highest score was taken in the patient activation subscale, and the lowest score was taken in the follow-up subscale.

Moreover, the quality of health care perceptions of independent patients was better.

Keywords: Chronic neurological disorders; Dependency; Health care quality; Nursing

\section{Introduction}

Many chronic diseases which are related to neurological system are considered as diseases that cause severe disability among individuals $[1,2]$. Stroke, multiple sclerosis (MS), and epilepsy all have an important place among these diseases. Stroke is one of the leading causes of morbidity and mortality all over the world [3]. According to the data in developed countries; stroke, which is most frequently encountered in neurological diseases, is ranked as the third after heart diseases and cancer as mortality cause and the first as a morbidity cause [4]. One other significant disease that causes functional inadequacy among individuals is MS [5]. This disease is ranked as the first among diseases that cause neurological disability in young adults regardless of trauma [6-8]. On the other hand, epilepsy is a disease that requires the individual to be prepared for physical changes, daily medication use, repeated doctor examinations, and acute medical emergencies which affect quality of life negatively [9].

Today, the purpose of healthcare for individuals with chronic neurological diseases is to increase the capacities of patients and their families to cope with problems, improve self-care, preserve and improve skills, meet information needs, and increase independence and quality of life [10].

The absence of an organized and developed care system may be listed among reasons for individuals with chronic diseases not receiving qualified and adequate care. This compels governments to make changes in health services and create a new chronic care system [11].

A chronic care model was developed by Wagner et al., in order to ensure quality in management of chronic diseases. This model is a model that is most frequently used in the management of chronic diseases and is considered the most effective [12,13], Though the chronic disease management was structured in accordance with the model; it is possible to restrict physiological and psychological effects of the disease, reduce the number of those applying to emergency units and their hospitalization, prevent dependence and increase quality of life $[14,15]$. With the help of patients consciously participating in their care, the disease is kept under control in a better way and a more effective joint work is maintained among medical team members.

Patient satisfaction constitutes a significant part of the quality in healthcare services. Improvement in results and quality of the care by planning appropriate treatment and follow-up brings the patient satisfaction as a consequence [16]. 
Expectations of patients from healthcare professionals may vary based on their age, gender, educational level, socio-cultural characteristics, dependence levels, and previous experiences with healthcare institutions [17]. While Glasgow and colleagues could not reveal significant differences in the PACIC scores regarding age, education, and gender [18,19]. Rosemann et al. [20] and Cramm et al. [21] found that younger, more highly educated patients report higher PACIC scores, indicating that their care aligns to a higher degree with the chronic care model.

Evaluation of quality in healthcare services affects the provision of a healthcare service of high quality. Therefore, it is considered necessary to recognize the perceptions of patients' of the given healthcare service and also examine factors affecting these perceptions. The purpose of this study therefore, is to assess chronic disease care in neurological diseases and assess the correlation between patients' dependence and the chronic disease care services.

\section{Methods}

\section{Design and sample}

The study is designed as a descriptive and cross-sectional one. It was conducted with 200 patients among those who applied to and who were registered in the neurology clinic of Ataturk University Hospital in Erzurum, Turkey between March 2014 to July 2014 these were those who met the research criteria. However, since 16 patients did not participate because they were either busy or unwilling, the study was completed with a total of 184 patients.

The inclusion criteria included (1) being diagnosed with Stroke, MS and Epilepsy six months prior to conducting the study, (2) being stayed at least 3 days to the neurology clinic and (3) being conscious. Patients with communication problems were excluded from the study.

All patients gave informed consent and the study was approved by Atatürk University Ethics Committee.

\section{Measures}

A three-part survey was used for data collection. The questionnaires included (1) Personal Information Form, (2) Patient Assessment of Chronic Illness Care (PACIC) scale, and (3) Barthell Index (BI).

\section{Individual information questionnaire}

The individual information questionnaire included age, sex, marital status, education, income status, place of residence, disease duration, co morbid conditions, hospital experience, and length of stay in hospital.

\section{Patient assessment of chronic illness care (PACIC)}

The initial version of the PACIC questionnaire was developed by Glasgow et al. [18]. Patients' assessed chronic illness care (PACIC) with a 20-item questionnaire comprising of five pre-defined subscales: patient activation (3 questions), delivery-system/practice design (3), goal setting/tailoring (5), problem solving/contextual (4), and followup/coordination (5). The five-point response scale ranged from 'almost never' to 'almost always' with higher scores indicating a more frequent presence of the respective aspect of chronic care. The PACIC score was the sum of participants' responses divided by 20 . Scores thus ranged from 1 to 5 with higher scores indicating a greater perception of involvement in self-management and receipt of chronic care counseling. Cronbach's alpha of patient activation $=0.83$, deliverysystem/practice design $=0.74$, goal setting/tailoring $=0.79$, problem solving/contextual $=0.86, \quad$ follow-up/coordination $=0.75$. Cronbach's alpha of the overall PACIC scale was 0.93 .

The reliability and the validity of the overall PACIC scale for the Turkish population have been demonstrated by Incirkus and Nahcivan [22]. In the original study, the Cronbach alfa value was calculated to be 0.91. In this study, Cronbach alfa parameter for overall PACIC was determined as 0.89 .

\section{Barthell index (BI)}

The BI is an assessment of a patient's functional level of independence in Activities of Daily Living (ADL) and is scored in increments of 5 points (highest possible total score $=100$ ). The values assigned to each item are weighed according to the amount of physical assistance required if the patient could not perform the activity independently. The BI measures 10 personal ADL related to self-care and mobility: control of bowels and bladder, grooming, toilet use, feeding, transfer, mobility, dressing, stairs and bathing. BI score ranges from 0 to 100 and lower scores indicate greater dependency [23].

The reliability and the validity of the BI for the Turkish population have been demonstrated by Kücükdeveciet al. [24]. In the original study, the Cronbach a value was calculated to be 0.93 . In this study, total Cronbach a parameter for BI was determined as 0.97 .

\section{Data collection}

The researcher visited the neurology polyclinic 3 days a week. Patients were assessed to determine whether they matched the inclusion criteria for the study. Data collection forms were applied to patients matching the criteria in face-to-face interview. Literate patients read and filled out the forms by themselves while illiterate patients $(n=30)$ filled out the forms with the help of the researcher who read the forms to them. Completion of data collection forms for each patient took 15-30 min.

\section{Statistical analysis}

Coding and statistical analyses of data were done by using the SPSS 18.0 package program. Percentage was used to evaluate the parameters of age, sex, education, marital status, income, place of residence, disease duration, comorbid conditions, hospital experience, length of stay in hospital and dependency level. Variance analysis was applied to examine the difference between the mean score of PACIC according to age, marital status, education level, place of residence and diagnosis of disease. The $t$ test was applied to determine differences between the mean quality of life scores according to gender, income status, comorbid conditions, hospital experience and length of stay in hospital. The Pearson's correlation analysis was applied to determine the relation between PACIC scores and point means of BI. Significance in all statistical analyses was defined as $\mathrm{P}<0.05$.

\section{Results}

Overall mean score of PACIC scale was found to be $3.05 \pm 0.57$. The lowest mean score was obtained in follow-up/coordination subscale $(2.73 \pm 0.86)$ among sub-scales of the scale, the highest mean score was found to be in patient activation subscale $(3.20 \pm 0.74)$ (Table 1). 
Citation: Dayapoglu N, Sahin ZA (2016) Assessment of Health Care Quality in Chronic Neurological Diseases: Stroke, Multiple Sclerosis and Epilepsy. J Neurol Neurophysiol 7: 402. doi:10.4172/2155-9562.1000402

Page 3 of 7

\begin{tabular}{|l|l|l|}
\hline Scale & Min-Max & X \pm SD \\
\hline Overall PACIC (0-5) & $1.70-4.65$ & $3.05 \pm 0.57$ \\
\hline PACIC subscales (0-5) & & \\
\hline Patient activation & $1.67-5.00$ & $3.20 \pm 0.74$ \\
\hline Delivery system design/decision support & $1.33-4.67$ & $3.15 \pm 0.75$ \\
\hline Goal setting/tailoring & $1.60-4.80$ & $3.13 \pm 0.64$ \\
\hline Problem solving/contextual & $1.50-5.00$ & $3.17 \pm 0.61$ \\
\hline Follow-up/coordination & $1.00-4.60$ & $2.73 \pm 0.86$ \\
\hline
\end{tabular}

$54.3 \%$ were primary education, $41.3 \%$ lived in the city and $54.3 \%$ was income equal to the expenses. $41.8 \%$ of patients applying to the neurology clinic had stroke, $70.1 \%$ did not have any comorbid disease, $65.2 \%$ had hospitalization experiences and $52.2 \%$ were staying in the hospital for 10 days and longer.

When mean scores of patients were evaluated according to age, the differences with respect to overall PACIC and all subscales of PACIC were found statistically significant $(\mathrm{P}<0.05)$ (Table 2$)$.

Patients who were married and high school and college graduates had higher patient activation and problem-solving mean scores among overall PACIC and the scale's sub-scale scores than the other groups and the difference between them was found to be statistically significant $(\mathrm{P}<0.05)$ (Table 2$)$.

The difference between gender, income level, and residence area and

Table 1: Score distribution of the PACIC among patients $(n=184)$ (PACIC-Patient Assessment of Chronic Illness Care; X: Mean; SD: Standard Deviation)

The mean age of patients was $49.41 \pm 21.83$ years; $45.7 \%$ were aged overall PACIC mean score was not statistically significant $(\mathrm{P}>0.05)$ (Table 2).

between 18 and 39 years; $52.7 \%$ were females, $56.0 \%$ were married,

\begin{tabular}{|c|c|c|c|c|c|c|c|}
\hline & & Patient Activation & $\begin{array}{l}\text { Delivery System } \\
\text { Design }\end{array}$ & Goal Setting & $\begin{array}{l}\text { Problem } \\
\text { Solving }\end{array}$ & $\begin{array}{l}\text { Follow } \\
\text { Coordination }\end{array}$ & Overall PACIC \\
\hline Characteristics & $\mathrm{n}$ & $X \pm S S$ & $X \pm S S$ & $X \pm S S$ & $X \pm S S$ & $X \pm S S$ & $X \pm S S$ \\
\hline \multicolumn{8}{|l|}{ Age (years) } \\
\hline $18-39$ & 84 & $3.50 \pm 0.73$ & $3.32 \pm 0.71$ & $3.28 \pm 0.64$ & $3.44 \pm 0.52$ & $2.90 \pm 0.99$ & $3.26 \pm 0.55$ \\
\hline $40-59$ & 32 & $3.02 \pm 0.70$ & $3.03 \pm 0.90$ & $3.00 \pm 0.64$ & $3.11 \pm 0.46$ & $2.68 \pm 0.74$ & $2.95 \pm 0.53$ \\
\hline \multirow[t]{2}{*}{60 and above } & 68 & $2.92 \pm 0.63$ & $3.00 \pm 0.69$ & $2.98 \pm 0.62$ & $2.84 \pm 0.62$ & $2.55 \pm 0.68$ & $2.84 \pm 0.54$ \\
\hline & & $F=14.494$ & $F=3.910$ & $F=4.954$ & $F=22.008$ & $F=3.139$ & $F=11.431$ \\
\hline P-value & & $P<0.001$ & $\mathrm{P}<0.05$ & $P<0.01$ & $P<0.001$ & $\mathrm{P}<0.05$ & $P<0.001$ \\
\hline \multicolumn{8}{|l|}{ Sex } \\
\hline Female & 97 & $3.209 \pm 0.81$ & $3.11 \pm 0.80$ & $3.10 \pm 0.62$ & $3.18 \pm 0.63$ & $2.78 \pm 0.88$ & $3.06 \pm 0.59$ \\
\hline \multirow[t]{2}{*}{ Male } & 87 & $3.206 \pm 0.66$ & $3.20 \pm 0.70$ & $3.14 \pm 0.67$ & $3.1 \pm 0.59$ & $2.68 \pm 0.83$ & $3.04 \pm 0.56$ \\
\hline & & $t=0.025$ & $\mathrm{t}=-0.769$ & $t=-0.393$ & $\mathrm{t}=0.458$ & $\mathrm{t}=0.807$ & $t=0.140$ \\
\hline P-value & & $P>0.05$ & $P>0.05$ & $P>0.05$ & $P>0.05$ & $P>0.05$ & $P>0.05$ \\
\hline \multicolumn{8}{|l|}{ Marital status } \\
\hline Married & 103 & $3.31 \pm 0.73$ & $3.23 \pm 0.73$ & $3.17 \pm 0.65$ & $3.25 \pm 0.57$ & $2.78 \pm 0.88$ & $3.12 \pm 0.56$ \\
\hline Single & 36 & $3.29 \pm 0.82$ & $3.13 \pm 0.83$ & $3.13 \pm 0.71$ & $3.34 \pm 0.63$ & $2.65 \pm 1.04$ & $3.10 \pm 0.64$ \\
\hline \multirow[t]{2}{*}{ Widow } & 45 & $2.89 \pm 0.62$ & $2.98 \pm 0.72$ & $3.00 \pm 0.58$ & $2.83 \pm 0.57$ & $2.60 \pm 0.61$ & $2.85 \pm 0.51$ \\
\hline & & $F=5.470$ & $F=1.788$ & $F=1.027$ & $F=9.691$ & $\mathrm{~F}=0.721$ & $F=3.714$ \\
\hline P-value & & $\mathrm{P}<0.01$ & $P>0.05$ & $P>0.05$ & $P<0.001$ & $P>0.05$ & $P>0.05$ \\
\hline \multicolumn{8}{|l|}{ Education } \\
\hline Illiterate & 30 & $2.81 \pm 0.65$ & $2.83 \pm 0.77$ & $2.95 \pm 0.54$ & $2.85 \pm 0.51$ & $2.57 \pm 0.73$ & $2.80 \pm 0.52$ \\
\hline Primary school & 100 & $3.18 \pm 0.73$ & $3.13 \pm 0.76$ & $3.13 \pm 0.61$ & $3.13 \pm 0.60$ & $2.76 \pm 0.80$ & $3.04 \pm 0.54$ \\
\hline \multirow[t]{2}{*}{ High school } & 54 & $3.46 \pm 0.71$ & $3.37 \pm 0.68$ & $3.21 \pm 0.74$ & $3.40 \pm 0.59$ & $2.77 \pm 1.01$ & $3.20 \pm 0.63$ \\
\hline & & $F=8.187$ & $F=5.262$ & $F=1.572$ & $F=8.556$ & $F=0.648$ & $F=4.937$ \\
\hline
\end{tabular}


Citation: Dayapoglu N, Sahin ZA (2016) Assessment of Health Care Quality in Chronic Neurological Diseases: Stroke, Multiple Sclerosis and

Page 4 of 7

\begin{tabular}{|c|c|c|c|c|c|c|c|}
\hline P-value & & $P<0.001$ & $P<0.01$ & $P>0.05$ & $P<0.001$ & $P>0.05$ & $P<0.01$ \\
\hline \multicolumn{8}{|l|}{ Income level } \\
\hline Income<expenditure & 89 & $3.12 \pm 0.75$ & $3.03 \pm 0.75$ & $3.06 \pm 0.59$ & $3.10 \pm 0.61$ & $2.72 \pm 0.81$ & $2.99 \pm 0.53$ \\
\hline \multirow[t]{2}{*}{ Income=expenditure } & 95 & $3.28 \pm 0.73$ & $3.27 \pm 0.74$ & $3.18 \pm 0.69$ & $3.22 \pm 0.61$ & $2.74 \pm 0.90$ & $3.11 \pm 0.61$ \\
\hline & & $t=-1.429$ & $\mathrm{t}=-2.233$ & $\mathrm{t}=-1.346$ & $t=-1.262$ & $\mathrm{t}=-0.203$ & $\mathrm{t}=-1.433$ \\
\hline P-value & & $P>0.05$ & $\mathrm{P}<0.05$ & $P>0.05$ & $P>0.05$ & $P>0.05$ & $P>0.05$ \\
\hline \multicolumn{8}{|l|}{ Place of residence } \\
\hline City & 76 & $3.28 \pm 0.80$ & $3.14 \pm 0.91$ & $3.08 \pm 0.74$ & $3.26 \pm 0.60$ & $2.80 \pm 0.96$ & $3.09 \pm 0.66$ \\
\hline Town & 71 & $3.26 \pm 0.65$ & $3.24 \pm 0.59$ & $3.21 \pm 0.58$ & $3.18 \pm 0.59$ & $2.76 \pm 0.85$ & $3.10 \pm 0.53$ \\
\hline \multirow[t]{2}{*}{ Village } & 37 & $2.94 \pm 0.74$ & $3.00 \pm 0.68$ & $3.03 \pm 0.54$ & $2.93 \pm 0.61$ & $2.54 \pm 0.61$ & $2.87 \pm 0.44$ \\
\hline & & $F=2.940$ & $F=1.237$ & $F=1.175$ & $\mathrm{~F}=3.847$ & $F=1.255$ & $\mathrm{~F}=2.291$ \\
\hline P-value & & $P>0.05$ & $P>0.05$ & $P>0.05$ & $P>0.05$ & $P>0.05$ & $P>0.05$ \\
\hline
\end{tabular}

Table 2: Comparison of PACIC scores related to socio-demographic characteristics of the patients $(\mathrm{n}=184)$. (PACIC: Patient Assessment of Chronic Illness Care; X: Mean; SD: Standard Deviation; p-value, differences between groups established with t Test or Variance analysis).

Mean scores of patients diagnosed with stroke in general PACIC, patient activation, and problem-solving sections were lower than those diagnosed with other neurological diseases and the difference between them was statistically significant $(\mathrm{P}<0.05)$ (Table 3$)$.

It was determined that patients with comorbid conditions are lower compared to patients with no comorbid conditions considering general
PACIC and goal determining, follow up mean score average for the lower dimensions of the scale which is also statistically significant $(\mathrm{P}<0.05)$ (Table 3).

In terms of patients' hospitalization experiences and hospitalization durations, a statistically significant difference was found between overall PACIC and all sub-scale mean scores $(\mathrm{P}<0.05)$ (Table 3$)$.

\begin{tabular}{|c|c|c|c|c|c|c|c|}
\hline Clinical characteristics & & Patient activation & $\begin{array}{c}\text { Delivery system } \\
\text { design }\end{array}$ & Goal setting & $\begin{array}{l}\text { Problem } \\
\text { solving }\end{array}$ & $\begin{array}{l}\text { Follow up/ } \\
\text { coordination }\end{array}$ & Overall PACIC \\
\hline & $\mathbf{n}$ & $X \pm S D$ & $X \pm S D$ & $X \pm S D$ & $X \pm S D$ & $X \pm S D$ & $X \pm S D$ \\
\hline \multicolumn{8}{|l|}{ Disease diagnosis } \\
\hline Stroke & 77 & $2.99 \pm 0.61$ & $3.06 \pm 0.68$ & $3.02 \pm 0.60$ & $2.89 \pm 0.59$ & $2.62 \pm 0.66$ & $2.90 \pm 0.52$ \\
\hline MS & 64 & $3.42 \pm 0.79$ & $3.25 \pm 0.77$ & $3.24 \pm 0.65$ & $3.42 \pm 0.52$ & $2.91 \pm 0.99$ & $3.22 \pm 0.57$ \\
\hline \multirow[t]{2}{*}{ epilepsy } & 43 & $3.27 \pm 0.80$ & $3.18 \pm 0.85$ & $3.14 \pm 0.70$ & $3.28 \pm 0.58$ & $2.66 \pm 0.93$ & $3.07 \pm 0.61$ \\
\hline & & $\mathrm{F}=6.238$ & $F=1.080$ & $F=1.993$ & $F=16.312$ & $F=2.232$ & $F=5.807$ \\
\hline P-value & & $P<0.01$ & $P>0.05$ & $P>0.05$ & $P<0.001$ & $P>0.05$ & $P<0.01$ \\
\hline \multicolumn{8}{|l|}{ Comorbid conditions } \\
\hline Yes & 55 & $3.13 \pm 0.75$ & $3.00 \pm 0.82$ & $2.92 \pm 0.66$ & $3.03 \pm 0.70$ & $2.48 \pm 0.77$ & $2.88 \pm 0.61$ \\
\hline \multirow[t]{2}{*}{ No } & 129 & $3.23 \pm 0.74$ & $3.22 \pm 0.72$ & $3.21 \pm 0.62$ & $3.22 \pm 0.56$ & $2.84 \pm 0.87$ & $3.12 \pm 0.55$ \\
\hline & & $\mathrm{t}=-0.818$ & $\mathrm{t}=-1.780$ & $t=-2.774$ & $t=-1.919$ & $t=-2.646$ & $t=-2.686$ \\
\hline P-value & & $P>0.05$ & $P>0.05$ & $P<0.01$ & $P>0.05$ & $P<0.01$ & $P<0.01$ \\
\hline \multicolumn{8}{|l|}{ Hospital experience } \\
\hline Yes & 120 & $3.09 \pm 0.74$ & $3.06 \pm 0.80$ & $3.09 \pm 0.69$ & $3.16 \pm 0.64$ & $2.63 \pm 0.90$ & $2.98 \pm 0.61$ \\
\hline No & 64 & $3.41 \pm 0.70$ & $3.33 \pm 0.61$ & $3.19 \pm 0.56$ & $3.17 \pm 0.56$ & $2.93 \pm 0.74$ & $3.18 \pm 0.49$ \\
\hline$P$-value & & $\mathrm{t}=-2.818$ & $t=-2.393$ & $t=-1.015$ & $t=-0.117$ & $t=-2.333$ & $t=-2.187$ \\
\hline
\end{tabular}


Page 5 of 7

\begin{tabular}{|l|c|c|c|c|c|c|c|}
\hline & & $P<0.01$ & $P<0.05$ & $P>0.05$ & $P>0.05$ & $P<0.05$ & $P<0.05$ \\
\hline Hospitalization duration & & & & & & \\
\hline 3- 9 days & 88 & $3.39 \pm 0.67$ & $3.43 \pm 0.63$ & $3.41 \pm 0.60$ & $3.29 \pm 0.62$ & $3.15 \pm 0.75$ & $3.32 \pm 0.56$ \\
\hline 10 days and over & 96 & $3.04 \pm 0.75$ & $2.89 \pm 0.77$ & $2.86 \pm 0.57$ & $3.05 \pm 0.58$ & $2.35 \pm 0.77$ & $2.80 \pm 0.47$ \\
\hline & & $\mathrm{t}=3.246$ & $\mathrm{t}=5.149$ & $\mathrm{t}=6.240$ & $\mathrm{t}=2.732$ & $\mathrm{t}=7.090$ & $\mathrm{t}=6.760$ \\
\hline P-value & & $\mathrm{P}<0.01$ & $\mathrm{P}<0.001$ & $\mathrm{P}<0.001$ & $\mathrm{P}<0.01$ & $\mathrm{P}<0.001$ & $\mathrm{P}<0.001$ \\
\hline
\end{tabular}

Table 3: Comparison of PACIC scores related to clinical variables of the patients $(n=184)$. (PACIC: Patient Assessment of Chronic Illness Care; X: Mean; SD: Standard Deviation; p-value, differences between groups established with $\mathrm{t}$ Test or Variance analysis).

The mean score for Barthell Index (BI) was $66.60 \pm 38.22$. When the distribution of independence levels of patients in their daily life activities was examined according to the $\mathrm{BI}, 34.2 \%$ were independent, $12 \%$ were mildly dependent, $15.8 \%$ were dependent on a medium level, $17.4 \%$ were severely dependent and $20.7 \%$ were dependent. There was a positive correlation between the BI and the areas of PACIC such as patient activation $(\mathrm{r}=0.439, \quad \mathrm{P}<0.01)$, delivery system design $(\mathrm{r}=0.407, \mathrm{P}<0.01)$, goal setting $(\mathrm{r}=0.331, \mathrm{P}<0.01)$, problem solving $(\mathrm{r}=0.489, \mathrm{P}<0.01)$, follow-up $(\mathrm{r}=0.230, \mathrm{P}<0.01)$ and overall PACIC $(\mathrm{r}=0.447, \mathrm{p}<0.01)$. This result showed that as independence levels of patients in their daily life activities increased, their healthcare qualities also increased (Table 4).

\begin{tabular}{|l|c|c|}
\hline \multirow{2}{*}{ PACIC Scale } & \multicolumn{2}{|l|}{ Barthell Index (BI) } \\
\cline { 2 - 3 } & $\mathbf{r}$ & $\mathbf{p}$ \\
\hline Patient activation & 0.439 & $\mathrm{p}<0.01$ \\
\hline Delivery system design/decision support & 0.407 & $\mathrm{p}<0.01$ \\
\hline Goal setting/tailoring & 0.331 & $\mathrm{p}<0.01$ \\
\hline Problem solving/contextual & 0.489 & $\mathrm{p}<0.01$ \\
\hline Follow up/coordination & 0.23 & $\mathrm{p}<0.01$ \\
\hline Overall PACIC & 0.447 & $\mathrm{p}<0.01$ \\
\hline
\end{tabular}

Table 4: Correlation between the PACIC scores with BI. (PACIC: Patient Assessment of Chronic Illness Care; BI: Barthell Index).

\section{Discussion}

Chronic conditions are increasingly becoming the primary concern of healthcare systems throughout the world and are soon to be the leading cause of disability [25]. Therefore, evaluation of the care provided for individuals with chronic neurological diseases is getting increasingly important. The results achieved as a result of this study conducted with the purpose of determining the healthcare quality of neurological diseases were discussed in accordance with relevant study results and literature knowledge.

The overall mean score obtained by patients from the PACIC scale within this study was $3.05 \pm 0.57$. When it is considered that the lowest score to be obtained from the scale is 0 and the highest score is 5 ; as their scores increase, chronic disease patients' levels of satisfaction from the healthcare services also increase and chronic disease management is adequate, it could be asserted that patients had an above-average level of satisfaction from the healthcare services. Similarly, Gensichen et al. [26] also found the overall PACIC scale mean score as $3.25 \pm 0.79$. Some studies in the literature have specified that patients have medium and above-medium levels of satisfaction from the healthcare services. The result of this study showed similarity with the results of previously conducted studies [19,22,27,28]. In addition, the lowest mean score among the sub-scales of PACIC scale was obtained in follow-up/coordination subscale $(2.73 \pm 0.86)$. This result was compatible with the results of the study conducted by Incirkus et al. [22].

In this study, we could not find any correlation between PACIC scale mean scores of patients in terms of the variables such as, patients' socio-demographic characteristics, gender, income level and residence area. Similarly, previous studies conducted on this subject also indicated that demographic characteristics such as gender, income level and residence area were not effective on healthcare satisfaction and on the adequacy of chronic disease management $[18,19,26,29,30]$.

We found that younger patients reported higher PACIC scores. As compatible with result of this study, Cramm and Nieboer [21] and Rosemannet al. [20] reported that younger patients had higher levels of satisfaction from the care they received as compared to elderly patients.

Higher levels of education were associated with higher scores with regard to overall PACIC, patient activation, and problem solving. Results of the study conducted by Rosemann et al. [20], were compatible with the result of this study and patients with higher educational backgrounds reported better views on healthcare services. This may be due to better coping skills and secured financial and social status of this group of patients. Several studies have shown that higher educational attainment is an important variable significantly associated with compliance with medical care [31,32]. When patients' marital statuses and their levels of satisfaction from healthcare were examined within the scope of this study, it was observed that married patients had higher mean scores in overall PACIC, patient activation and problem-solving sections. Thi et al. [33] also, reported that married patients were more satisfied with hospital services. This result was in parallel with our results. This result may be explained by the fact that married patients had lower care needs depending on the social support they received from their spouses and children and therefore, their levels of expectations from medical professionals reduced.

When healthcare quality was analyzed in terms of patients' disease types; total PACIC, patient activation and problem-solving mean scores of stroke patients were found to be lower. As stroke patients staying in the neurology clinic were more dependent in their daily life 
activities, their expectation levels were also considered to be higher. In the study conducted by Mollalogluet al. [34], to evaluate the disability in neurological diseases, they found that stroke patients experienced mobility-related disability in more serious levels.

In this study; general PACIC, objective-setting and follow-up mean scores of patients with co morbid disease were found to be lower. In line with the result of this study, Gensichen et al. [26], found a negative correlation between the number of additional physical conditions and "patient activation" among the PACIC scale's subscales.

We found that patients with no previous hospital experiences had better views on care services. Similar result was also reported in the study of Yilmaz [35]. This result may be interpreted in a way that individuals who were not previously hospitalized did not interact with medical care service providers therefore, their expectations remained limited.

It was found that as patients' hospitalization duration extended, their general PACIC and sub-scale mean scores decreased. As compatible with result of this study, Adams et al. [36], also found the chronic care model components of patients hospitalized for longer periods to be lower.

When the distribution of patients' independence levels was examined in terms of their daily life activities according to the Barthell Index (BI), it was interesting to note that only $34.2 \%$ of individuals with chronic neurological diseases were independent in maintaining their daily life activities. It has also been determined in similar previous studies that the majority of patients become dependent on others in maintaining their daily life activities during the course of the disease [34,37]. Many of the chronic diseases related to neurological system are considered diseases that cause severe restrictions and inadequacy for the individual.

We found a positive correlation between all sub-scales of BI and PACIC scale in this study. According to this result, as independence level increased in patients' daily life activities, the healthcare quality also increased. Although several parameters that could affect the quality of the healthcare were evaluated in previously conducted studies; no studies analyzing the correlation between patients' independence level and healthcare satisfaction have been found in the literature. When this result was compared with the findings of studies that examine the burden of care providers and their care quality perceptions according to patients' dependence levels; a positive correlation was reported between patients' dependence levels in their daily life activities and the burden of care [38,39].

\section{Conclusion}

According to the result of this study, individuals with neurological disease were found to have a medium level of satisfaction of healthcare. Medical professionals should ensure that they apply chronic disease models on patients effectively, in order to keep the healthcare quality at a desired level among individuals with neurological diseases. In parallel with this apprehension, it is a major necessity to make arrangements that focus on applying chronic care models in order to increase satisfaction among patients in terms of the healthcare services in hospitals.

We also found a positive correlation between the independence levels of patients in their daily life activities and the healthcare quality. This result showed that as patients' independence level increased, the quality of healthcare also increased. Therefore, a dynamic symptom management plan that is suitable with the patient's dependence status should be developed.

Our findings may have important implications towards the care of neurological disease. Further research is needed to confirm our results and assess possible implications for implementing the chronic care model in neurological diseases.

\section{Acknowledgement}

The authors thank the nurses, directors and individuals with stroke, MS, epilepsy of the neurology clinics at Ataturk University Hospital for their help with this study.

\section{Competing Interests}

The authors of this paper have no conflicts of interest to report. All authors have approved the final draft of the article.

\section{Authors' Contributions}

DN; conceived, designed and did data collection, did statistical analysis manuscript writing. SZA; editing of manuscript. All authors read and approved the final manuscript.

\section{Disclaimer}

This article was presented in the poster session at 16th National internal Medicine Congress (15-19 October 2014 Antalya/Turkey).

\section{References}

1. Crawford DK, Mangiardi M, Xia X, López-Valdés HE, Tiwari-Woodruff SK (2009) Functional recovery of callosal axons following demyelination: A critical window. Neuroscience 164: 1407-1421.

2. Kalb R (2007) The emotional and psychological impact of multiple sclerosis relapses. J Neurol Sci 256 Suppl 1: S29-33.

3. Caulfield AF, Wijman CA (2008) Management of acute ischemic stroke. Neurol Clin 26: 345-371.

4. Warlow CP, van Gijn J, Dennis MS, Wardlaw JM, Bamford JM, et al. (2008) Stroke: A practical guide to management. (3rd Edn), Oxford: Blackwell Publishing, USA.

5. Mollaoglu M (2012) Multiple sclerosis and care. In: Durna Z, Ed: Chronic illness and care. Istanbul: Nobel medicine pp: 261-276.

6. Ozdemir G (2003) Neurological Diseases. In: Iliçin G, Biberoglu K, Süleymanlar G, Ünal S, eds. Internal medicine. Ankara: Günes publishing, pp: 3609-3636.

7. Vercellino M, Masera S, Lorenzatti M, Condello C, Merola A, et al. (2009) Demyelination, inflammation and neurodegeneration in multiple sclerosis deep gray matter. J Neuropathol Exp Neurol 68: 489-502.

8. Forbes A, While A, Mathes L, Griffths P (2006) Health problems and health-related quality of life in people with multiple sclerosis. Clin Rehabil 20: 67-78.

9. de Boer HM (2010) Epilepsy stigma: moving from a global problem to global solutions. Seizure 19: 630-636.

10. Wagner EH (2000) The role of patient care teams in chronic disease management. BMJ 320: 569-572.

11. Cumbie SA, Conley VM, Burman ME (2004) Advanced practice nursing model for comprehensive care with chronic illness: model for promoting process engagement. ANS 27: 70-80.

12. Beaglehole R, Epping-Jordan J, Patel V, Chopra M, Ebrahim S, et al. (2008) Improving the prevention and management of chronic disease in low-income and middle-income countries: A priority for primary health care. Lancet 372: 940-949. 
Citation: Dayapoglu N, Sahin ZA (2016) Assessment of Health Care Quality in Chronic Neurological Diseases: Stroke, Multiple Sclerosis and Epilepsy. J Neurol Neurophysiol 7: 402. doi:10.4172/2155-9562.1000402

Page 7 of 7

13. Piatt GA, Zgibor JC (2007) Novel approaches to diabetes care: a population perspective. Curr Opin Endocrinol Diabetes Obes 14: 158-165.

14. Haskett $\mathrm{T}$ (2006) Chronic illness management: Changing the system. Home Health Care Management Prac 18: 492-496.

15. Bodenheimer T, Wagner EH, Grumbach K (2002) Improving primary care for patients with chronic illness. JAMA 288: 1775-1779.

16. Rothman AA, Wagner EH (2003) Chronic illness management: What is the role of primary care? Ann Intern Med 138: 256-261.

17. Kavuncubasi S (2000) Hospital and Health Institutions Management. Ankara: Political publishing, pp: 292-295.

18. Glasgow RE, Wagner EH, Schaefer J, Mahoney LD, Reid RJ, et al. (2005) Development and validation of the Patient Assessment of Chronic Illness Care (PACIC). Med Care 43: 436-444.

19. Glasgow RE, Whitesides H, Nelson CC (2005) Use of the patient assessment of chronic illness care (PACIC) with diabetic patients: relationship to patient characteristics, receipt of care and selfmanagement. Diabetes Care 28: 2655-2661.

20. Rosemann T, Laux G, Szecsenyi J, Grol R (2008) The chronic care model: Congruency and predictors among primary care patients with osteoarthritis. Qual Saf Health Care 17: 442-446.

21. Cramm JM, Nieboer AP (2012) The chronic care model: congruency and predictors among patients with cardiovascular diseases and chronic obstructive pulmonary disease in the Netherlands. BMC 12: 242.

22. Incirkus K, Nahcivan NÖ (2011) Validity and reliability study of Turkish version of the patient assessment of chronic illness care-patient form. Deuhyo ED 4: 102-109.

23. Mahoney FI, Barthel DW (1965) Functional evaluation: The Barthel Index. Md State Med J 14: 61-65.

24. Küçükdeveci AA, Yavuzer G, Tennant A, Süldür N, Sonel B, et al. (2000) Adaptation of the modified Barthel Index for use in physical medicine and rehabilitation in Turkey. Scand J Rehabil Med 32: 87-92.

25. Epping-Jordan JE, Pruitt SD, Bengoa R, Wagner EH (2004) Improving the quality of health care for chronic conditions. Qual Saf Health Care 13: 299-305.

26. Gensichen J, Serras A, Paulitsch MA, Rosemann T, König J, et al. (2011) The patient assessment of chronic illness care questionnaire: Evaluation in patients with mental disorders in primary care. Community Ment Health J 47: 447-453

27. Aytar G, Yesildal N (2004) Patient satisfaction in clinic departments. Düzce Tip Fak Derg 3: 10-14.

28. Larrabee JH, Ostrow CL, Withrow ML, Janney MA, Hobbs GR Jr, et al. (2004) Predictors of patient satisfaction with inpatient hospital nursing care. Res Nurs Health 27: 254-268.

29. Geberemichael SG, Metaferia GZ, Takele GM, Johnston JC (2011) Patient satisfaction with outpatient neurology services: A momentum for improvement. J Neurol Sci 303: 128-132.

30. Lochoro $\mathrm{P}$ (2004) Measuring patient satisfaction in UCMB health instituions. Health Policy and Develop 2: 243-248.

31. Unzueta M, Globe D, Wu J, Paz S, Azen S, et al. (2004) Compliance with recommendations for follow-up care in Latinos: the Los Angeles Latino Eye Study. Ethn Dis 14: 285-291.

32. Rahman SM, Dignan MB, Shelton BJ (2003) Factors influencing adherence to guidelines for screening mammography among women aged 40 years and older. Ethn Dis 13: 477-484.

33. Nguyen Thi PL, Briançon S, Empereur F, Guillemin F (2002) Factors determining inpatient satisfaction with care. Soc Sci Med 54: 493-504.

34. Mollaoglu M, Fertelli TK, Tuncay FÖ (2011) Disability in elderly patients with chronic neurological illness: Stroke, multiple sclerosis and epilepsy. Arch GerontolGeriatr 53: e227-231.

35. Yilmaz M (2001) A measure of health care quality: patient satisfaction. Cumhuriyet Univ Hems YO Derg 5: 69-74.

36. Adams SG, Smith PK, Allan PF, Anzueto A, Pugh JA, et al. (2007) Systematic review of the chronic care model in chronic obstructive pulmonary disease prevention and management. Arch Intern Med 167: 551-561.

37. Einarsson U, Gottberg K, Fredrikson S, von Koch L, Holmqvist LW (2006) Activities of daily living and social activities in people with multiple sclerosis in Stockholm County. Clin Rehabil 20: 543-551.

38. Mollaoglu M, Tuncay ÖF, Fertelli KT (2011) Care burden of care givers of stroke patients and related factors. Deuhyo Ed 4: 125-130.

39. Kalav S (2011) The relationship between caregiving burden and quality of life in family caregivers of stroke patients [dissertation]. University of Mersin. 